\title{
Nonlinear Frequency Response Analysis as a Tool for Identification of Adsorption Kinetics: Case Study_Pore-Surface Diffusion Control
}

\author{
Danica V. Brzić (D) and Menka T. Petkovska \\ Department of Chemical Engineering, Faculty of Technology and Metallurgy, University of Belgrade, 11000 Belgrade, Serbia \\ Correspondence should be addressed to Danica V. Brzić; danica@tmf.bg.ac.rs
}

Received 30 December 2018; Revised 27 March 2019; Accepted 4 April 2019; Published 23 May 2019

Academic Editor: Sergey A. Suslov

Copyright (C) 2019 Danica V. Brzić and Menka T. Petkovska. This is an open access article distributed under the Creative Commons Attribution License, which permits unrestricted use, distribution, and reproduction in any medium, provided the original work is properly cited.

\begin{abstract}
In the present paper, the Nonlinear Frequency Response (NFR) analysis is applied for theoretical study of kinetics of adsorption governed by pore-surface diffusion. The concept of higher-order frequency response functions (FRFs) is used. Based on a nonlinear mathematical model for adsorption of pure gas and spherical adsorbent particles, the theoretical first- and second-order FRFs, which relate the adsorbate concentration in the particle to the surrounding pressure $\left(F_{1}(\omega)\right.$ and $\left.F_{2}(\omega, \omega)\right)$, have been derived. The obtained FRFs have been simulated for different steady-state pressures and different ratios (between zero and one) of surface to pore diffusion coefficients. The results show that, unlike $F_{1}(\omega), F_{2}(\omega, \omega)$ exhibits features which unambiguously distinguish the pore-surface diffusion model from pure pore diffusion and micropore diffusion. Based on the characteristic features of $F_{1}(\omega)$ and $F_{2}(\omega, \omega)$, a new methodology for direct estimation of the separate values of the pore and surface diffusion coefficients has been established.
\end{abstract}

\section{Introduction}

Although the processes in chemical engineering are mostly nonlinear in nature, it has been a common practice in chemical engineering to use linear tools for analysis of those processes, providing thus significant simplifications on the cost of limited applicability. However, modern trends of design, which include intensification and optimization methods, imply the necessity of implementation of rigorous procedures and nonlinear mathematical tools.

Adsorption processes are one of the examples of nonlinear processes with complex dynamic behavior. If we consider adsorption of a gas on a porous adsorbent bead, then the overall rate of the process may be controlled by the following individual processes: mass transfer through the fluid film around the particle, macropore diffusion, surface diffusion, micropore diffusion, and adsorption/desorption [1]. Since the intrinsic rate of the adsorption/desorption on the active site is usually fast, the overall rate is usually controlled by diffusional resistances. Depending on the properties of the adsorbate/adsorbent system and operating conditions, relative contributions of individual resistances may vary. Reliable identification of relative dominancy of the individual resistances is still a challenging issue. In the literature, a number of methods for measuring diffusion coefficients in solid adsorbents have been reported: uptake rate [2,3], piezometric method [4], chromatographic method [5], infrared spectroscopy [6], vacuum temperature-programmed desorption (TPD) [7], electrochemical impedance spectroscopy (EIS) [8], combination of TPD and EIS [9], frequency response (FR) method [10-15], and temperature FR method [16].

Since the FR method uses the response of the system to sine-wave excitations, it introduces additional degree of freedom (frequency of the sine-wave) compared to the step and pulse response techniques, which makes the FR method advantageous regarding distinguishing individual time constants of multikinetic processes [17].

Adsorption of gases in commercial porous adsorbents is often controlled by pore-surface diffusion, where pore and surface diffusion take place in parallel and their rates 
are characterized by pore diffusion coefficient $\left(D_{p}\right)$ and surface diffusion coefficient $\left(D_{s}\right)$. Classical FR method, which uses the small amplitudes of input oscillations in order to obtain the first-order (linear) frequency response function (FRF), fails to identify the pore-surface diffusion mechanism. Namely, the first-order FRFs for micropore diffusion, macropore diffusion, and pore-surface diffusion have the same shapes [18] making the distinguishing between them impossible. For the pore-surface diffusion mechanism, the linear FR allows estimation of only an effective diffusion coefficient, $D_{\text {eff }}[18]$, but not the separate values of $D_{p}$ and $D_{s}$.

The pioneering work of Petkovska and Do [19] has made a step forward in adsorption kinetic studies by extending the linear FR to the nonlinear range. They considered the nonlinear response of the system (for larger input amplitude excitations) and used the Volterra-Weiner concept of higherorder FRFs in order to obtain the first-, second-, and thirdorder FRFs for nonlinear adsorption models. Their early results for single mechanism models (Langmuir, film resistance control, and micropore diffusion) [20], which showed that the second-order FRFs for different mechanisms had different shapes, indicated the potential of the nonlinear FR analysis for identification of the kinetic mechanism, based on the pattern of the second-order FRF. In the later work, the derivation of FRFs up to the second-order for more complex models (micropore-macropore diffusion with and without film resistance) confirmed that the secondorder FRFs have enough specific features for distinguishing between different mechanisms [21]. The methodology was also applied for some cases of nonisothermal adsorption (nonisothermal micropore diffusion [22] and nonisothermal macropore diffusion [23]). A procedure for estimating the equilibrium and kinetic parameters was also developed [18, 22, 23].

In practical applications of the nonlinear frequency response (NFR) approach, the identification of the kinetic mechanism is done by comparison of the experimental second-order FRF with the theoretical ones corresponding to different kinetic models. The procedure for obtaining the experimental FRFs up to the second-order has already been established [24, 25] and validated [26]. The broader exploiting of the NFR concept is partially limited by the fact that derivation of the theoretical second-order FRF for complex kinetic model may become rather tedious and that sometimes no analytical solution for the second-order FRF can be obtained. However, the FRFs need to be derived only once and then can be recalled from the library whenever needed. The existing library of theoretical FRFs comprises mostly the FRFs for plane geometry of the adsorbent particles, while the FRFs for the spherical geometry, which is, in many cases, much better approximation of the real adsorbent shape, are strongly needed.

The aim of this work was to check the ability of the NFR concept for identification and characterization of the pore-surface diffusion control kinetics, for spherical particles. Firstly, the FRFs up to the second-order for the nonlinear pore-surface diffusion model for spherical particle geometry have been derived. Numerical simulations of the FRFs for different parameters were employed in order to recognize the characteristic features for model identification. Finally, the methodology for estimation of the separate values of the pore and surface diffusion coefficients has been established.

\section{Nonlinear Frequency Response and Concept of Higher-Order FRFs}

Since the Volterra-Weiner concept of higher-order FRFs [27] will be used for derivation of the FRFs, it will be briefly presented. This concept represents the generalization of the well-known approach of convolutional integral and definition of the FRF, used in the linear frequency domain analysis. Namely, the dynamic response of a stable linear single-input single-output system to an arbitrary input signal $x(t)$ can be defined as a convolution integral:

$$
y^{l i n}(t)=\int_{-\infty}^{\infty} g(\tau) x(t-\tau) d \tau
$$

where $g(\tau)$ represents the impulse-response function of the system, or its kernel. By taking into account the definition of FRF [27]:

$$
G(\omega)=\int_{-\infty}^{\infty} g(\tau) e^{-j \omega \tau} d \tau
$$

it is possible to relate the time domain response of the system, $y^{l i n}(t)$, with its FRF $G(\omega)$ (which is the complex function of a single variable-frequency). For the input in the form of periodic function $x(t)=A \mathrm{e}^{j \omega t}$, the response defined by Eq. (1) becomes

$$
y^{l i n}(t)=A e^{j \omega t} \int_{-\infty}^{\infty} g(\tau) e^{-j \omega \tau} d \tau
$$

which, considering definition (2), becomes

$$
y^{l i n}(t)=A e^{j \omega t} G(\omega)
$$

When a nonlinear system with polynomial nonlinearities is the subject of the same arbitrary input $x(t)$, the response may be represented as an indefinite sum of multidimensional convolutional integrals (Volterra series):

$$
\begin{aligned}
& y^{\text {nonlin }}(t)=\sum_{n=1}^{\infty} y_{n}(t) \\
& y_{n}(t) \\
& =\int_{-\infty}^{\infty} \cdots \int_{-\infty}^{\infty} g_{n}\left(\tau_{1}, \cdots, \tau_{n}\right) \prod_{i=1}^{n} x\left(t-\tau_{i}\right) d \tau_{1} \cdots d \tau_{n}
\end{aligned}
$$

where $g_{n}\left(\tau_{1}, \ldots, \tau_{\mathrm{n}}\right)$ is the generalized impulse response function of order $n$, or $n$-th order Volterra kernel. The first element of the series, $y_{1}(t)$, has the same form as $y^{\text {lin }}(t)$ (Eq. (1)) and represents the response of the linearized system, while each of the higher terms $(n>1)$ represents the contribution of the nonlinearities of $n$-th order.

In analogy to the Fourier transform of the linear system's kernel, which defines the FRF of a linear system (Eq. (2)), 
the multidimensional Fourier transform of the $n$-th order Volterra kernel defines the generalized $n$-th order FRF:

$$
\begin{aligned}
& G_{n}\left(\omega_{1}, \cdots, \omega_{n}\right) \\
& =\int_{-\infty}^{\infty} \cdots \int_{-\infty}^{\infty} g_{n}\left(\tau_{1}, \cdots, \tau_{n}\right) e^{-j\left(\omega_{1} \tau_{1}+\cdots \omega_{n} \tau_{n}\right)} d \tau_{1} \cdots d \tau_{n}
\end{aligned}
$$

which is a complex function of $n$ frequencies. In order to establish the relation between the response of the nonlinear system defined by Eqs. (5) and (6) and the FRFs of the different orders, defined by Eq. (7), we will consider the input periodic function $x(t)$ in the form

$$
x(t)=A_{1} e^{j \omega_{1} t}+A_{2} e^{j \omega_{2} t}
$$

By expanding the Volterra series (Eqs. (5) and (6)) the response will be

$$
y(t)=y_{1}(t)+y_{2}(t)+\ldots
$$

where

$$
\begin{aligned}
y_{1}(t)= & G_{1}\left(\omega_{1}\right) A_{1} e^{j \omega_{1} t}+G_{1}\left(\omega_{2}\right) A_{2} e^{j \omega_{2} t} \\
y_{2}(t)= & G_{2}\left(\omega_{1}, \omega_{1}\right) A_{1}^{2} e^{2 j \omega_{1} t} \\
& +2 G_{2}\left(\omega_{1}, \omega_{2}\right) A_{1} A_{2} e^{j\left(\omega_{1}+\omega_{2}\right) t} \\
& +G_{2}\left(\omega_{2}, \omega_{2}\right) A_{2}^{2} e^{2 j \omega 2 t}
\end{aligned}
$$

The first element $y_{1}(t)$ contains two terms, which are responses of the linearized system to the individual harmonics contained in the input (Eq. (8)). However, the second element of the response, $y_{2}(t)$, contains the nonlinearities of the second order, which are second order interactions of the individual harmonics $\left(\left(e^{j \omega_{1} t}\right)^{2}\right.$ and $\left(e^{j \omega_{2} t}\right)^{2}$, the first and the third terms in Eq. (11)) and second order intermodulations (combined effect) of two input harmonics $\left(e^{j \omega_{1} t} \times e^{j \omega_{2} t}\right.$, the second term in Eq. (11)). Multiple interactions and intermodulations of input frequencies are characteristic only for the nonlinear systems.

\section{Derivation of the FRFs for Pore-Surface Diffusion Model}

3.1. Model Equations. For the derivation of the first- and second-order FRFs, the general procedure for derivation of theoretical higher-order FRFs [28] based on Volterra-Weiner approach is followed. According to that procedure, the first step is setting up the nonlinear mathematical model of the adsorption process. For isothermal pore-surface diffusion control, the mass balance over the adsorbing porous particle (of the radius $\mathrm{R}$ ) is given by the following equation:

$$
\begin{aligned}
(1-\varepsilon) \frac{\partial q_{i}}{\partial t}+\varepsilon_{p} \frac{\partial c_{i}}{\partial t}= & (1-\varepsilon) \frac{1}{r^{\sigma}} \frac{\partial}{\partial r}\left(D_{s} r^{\sigma} \frac{\partial q_{i}}{\partial r}\right) \\
& +\varepsilon_{p} \frac{1}{r^{\sigma}} \frac{\partial}{\partial r}\left(D_{p} r^{\sigma} \frac{\partial c_{i}}{\partial r}\right)
\end{aligned}
$$

where $q_{i}$ and $c_{i}$ are the sorbate concentrations in the solid phase and in the pore, respectively, both defined as relative deviations from the corresponding steady-state values:

$$
\begin{aligned}
& q_{i}(r)=\frac{Q_{i}(r)-Q_{i s}}{Q_{i s}} \\
& c_{i}(r)=\frac{C_{i}(r)-C_{i s}}{C_{i s}}
\end{aligned}
$$

$\varepsilon$ is porosity of the particle and $\varepsilon_{p}$ is the modified porosity:

$$
\varepsilon_{p}=\varepsilon \frac{C_{i s}}{Q_{i s}}
$$

$D_{p}$ is pore diffusion coefficient, $D_{s}$ is surface diffusion coefficient, and $\sigma$ is the shape factor $(\sigma=2$ for spherical geometry). The subscript $s$ denotes the values in the steadystate. The boundary conditions are based on the assumptions of concentration profiles symmetry

$$
r=0: \quad \frac{\partial c_{i}}{\partial r}=\frac{\partial q_{i}}{\partial r}=0
$$

and negligible film resistance

$$
r=R: \quad c_{i}=p
$$

where $\mathrm{p}$ is the dimensionless pressure, or sorbate concentration in the gas phase, defined as

$$
p=\frac{P-P_{s}}{P_{s}}
$$

Further, local equilibrium within the pore is assumed, so $q_{i}$ and $c_{i}$ are related by the adsorption isotherm relation:

$$
q_{i}=f\left(c_{i}\right)
$$

which is generally nonlinear. The adsorption isotherm relation (19), as a source of nonlinearity, is expressed in the form of Taylor series around the steady state:

$$
q_{i}\left(c_{i}\right)=a c_{i}+b c_{i}^{2}+\ldots
$$

where $a$ and $b$ are proportional to the first and second derivatives of the adsorption isotherm, respectively (their definitions are given in Table 1). Eq. (20) is substituted into Eq. (12), and the resulting PDE has only one dependent variable, $c_{i}$.

The overall adsorbate concentration within the particle at position $r, q(r)$, is defined as

$$
q(r)=\frac{\varepsilon_{p}}{\varepsilon_{p}+(1-\varepsilon)} c_{i}(r)+\frac{(1-\varepsilon)}{\varepsilon_{p}+(1-\varepsilon)} q_{i}(r)
$$

and the mean concentration in the adsorbent particle $\langle q\rangle$ is given as

$$
\langle q\rangle=\frac{\sigma+1}{R^{\sigma+1}} \int_{0}^{R} r^{\sigma} q(r) d r
$$


TABLE 1: Definitions of isotherm coefficients.

\begin{tabular}{|c|c|c|c|}
\hline$a=\frac{\partial q_{i}}{\partial c_{i}}$ & $b=\frac{1}{2} \frac{\partial^{2} q_{i}}{\partial c_{i}^{2}}$ & $a_{e f f}=\frac{\varepsilon_{p}+(1-\varepsilon) a}{\varepsilon_{p}+(1-\varepsilon)}$ & $b_{e f f}=\frac{(1-\varepsilon) b}{\varepsilon_{p}+(1-\varepsilon)}$ \\
\hline
\end{tabular}

3.2. Definitions of FRFs. In order to define the FRFs corresponding to the pore-surface diffusion model defined in the previous section, we need to define the input and output variables. Since we consider the model on the particle scale, the input is the dimensionless pressure $p$ and the output is the dimensionless mean concentration in the adsorbent particle $\langle q\rangle$, defined in $\mathrm{Eq}$ (22). Consequently, we define the main set of FRFs $F_{1}(\omega), F_{2}(\omega, \omega), \ldots$, which relate $<q>$ to $p$. However, since $\langle q\rangle$ depends on $q(r)$ (Eq. (22)) and consequently on $c_{i}(r)$ (Eq. (21)), we need to define two auxiliary sets of FRFs: $F_{1}{ }^{*}(\omega), F_{2}{ }^{*}(\omega, \omega), \ldots$, which relate $q(r)$ to $p$ and $H_{1}{ }^{*}(\omega)$, $H_{2}{ }^{*}(\omega, \omega), \ldots$, which relate $c_{i}(r)$ to $p$.

3.3. First-Order FRF. In order to derive the first-order FRF, $F_{1}(\omega)$, the input variable $(p)$ is expressed as a single harmonic periodic function:

$$
p=A e^{j \omega t}
$$

and the output variable $c_{i}(r, t)$ is expressed as a response according to Eq. (4):

$$
c_{i}(r, t)=A e^{j \omega t} H_{1}^{*}(\omega)
$$

The input and output relations (Eqs. (23) and (24)) are substituted into the model equations (12) to (22). By equalizing the coefficients of $A e^{j \omega t}$ on each side of the model equations the following ODE in which $H_{1}^{*}(\omega)$ is the dependent variable is obtained:

$$
\frac{d^{2} H_{1}^{*}(\omega, r)}{d r^{2}}+\frac{2}{r} \frac{d H_{1}^{*}(\omega, r)}{d r}-\frac{j \omega}{D_{e f f}} H_{1}^{*}(\omega, r)=0
$$

where

$$
D_{e f f}=\frac{(1-\varepsilon) a D_{s}+\varepsilon_{p} D_{p}}{(1-\varepsilon) a+\varepsilon_{p}}
$$

with boundary conditions

$$
\begin{array}{ll}
r=0 & \frac{d H_{1}^{*}(\omega, r)}{d r}=0 \\
r=R & H_{1}^{*}(\omega, r)=1
\end{array}
$$

Equation (25) is a second-order homogeneous ODE and has the following analytical solution:

$$
H_{1}^{*}(\omega, r)=\frac{R}{r} \frac{\sinh \left(r \sqrt{j \omega / D_{\text {eff }}}\right)}{\sinh \left(R \sqrt{j \omega / D_{\text {eff }}}\right)}
$$

Using Eq. (21) the function $F_{1}{ }^{*}(\omega, r)$ is obtained:

$$
F_{1}^{*}(\omega, r)=a_{e f f} H_{1}^{*}(\omega, r)
$$

where $a_{e f f}$ is the effective first-order concentration coefficient of the adsorption isotherm, defined in Table 1. Finally, the first-order FRF with respect to the mean concentration in the particle $\langle q\rangle$ is obtained using Eq. (22):

$$
F_{1}(\omega)=a_{e f f} \frac{3}{R^{2}} \frac{R \sqrt{j \omega / D_{e f f}} \cdot \operatorname{ctgh}\left(R \sqrt{j \omega / D_{e f f}}\right)-1}{j \omega / D_{\text {eff }}}
$$

3.4. Second-Order FRF. In order to derive the second-order FRF, the input variable $(p)$ is expressed as a sum of two harmonics of different frequencies $\left(\omega_{1}, \omega_{2}\right)$ :

$$
p=A_{1} e^{j \omega_{1} t}+A_{2} e^{j \omega_{2} t}
$$

and the output variable $c_{i}(r, t)$ is expressed according to Eqs. (9), (10), and (11):

$$
\begin{aligned}
c_{i}(r, t)= & H_{1}^{*}\left(\omega_{1}\right) A_{1} e^{j \omega_{1} t}+H_{1}^{*}\left(\omega_{2}\right) A_{2} e^{j \omega_{2} t} \\
& +H_{2}^{*}\left(\omega_{1}, \omega_{1}\right) A_{1}^{2} e^{2 j \omega_{1} t} \\
& +2 H_{2}^{*}\left(\omega_{1}, \omega_{2}\right) A_{1} A_{2} e^{j\left(\omega_{1}+\omega_{2}\right) t} \\
& +H_{2}^{*}\left(\omega_{2}, \omega_{2}\right) A_{2}^{2} e^{2 j \omega_{2} t}
\end{aligned}
$$

The input and output relations (Eqs. (32) and (33)) are substituted into the model equations (12) to (22). By equalizing the coefficients of $A_{1} A_{2} e^{j\left(\omega_{1}+\omega_{2}\right) t}$ on each side of the model equations, the following expression for $H_{2}^{*}\left(\omega_{1}, \omega_{2}\right)$, for the case $\omega_{1}=\omega_{2}$, is obtained:

$$
\begin{gathered}
\frac{d^{2} H_{2}^{*}(\omega, \omega, r)}{d r^{2}}+\frac{2}{r} \frac{d H_{2}^{*}(\omega, \omega, r)}{d r}-\frac{2 j \omega}{D_{e f f}} H_{2}^{*}(\omega, \omega, r) \\
=2 f b j \omega H_{1}^{*^{2}}(\omega, r)-\frac{4}{r} f b D_{s} H_{1}^{*}(\omega, r) \frac{d H_{1}^{*}(\omega, r)}{d r} \\
-2 f b D_{s}\left(1+H_{1}^{*}(\omega, r)\right) \frac{d^{2} H_{1}^{*}(\omega, r)}{d r^{2}}
\end{gathered}
$$

where parameter $f$ is defined:

$$
f=\frac{(1-\varepsilon)}{(1-\varepsilon) a D_{s}+\varepsilon_{p} D_{p}}
$$

with boundary conditions

$$
\begin{array}{ll}
r=0 & \frac{d H_{2}^{*}(\omega, \omega, r)}{d r}=0 \\
r=R & H_{2}^{*}(\omega, \omega, r)=0
\end{array}
$$

By incorporating Eq. (29) for $H_{1}^{*}(\omega, r)$ into Eq. (34), as well as the expressions for first and second derivatives 
TABLE 2: Adsorption isotherm coefficients at 298K [29].

\begin{tabular}{lccc}
\hline$P_{\mathrm{s}}$ (mbar) & 20 & 40 & 75 \\
\hline$a$ & 0.4369 & 0.3684 & 0.3105 \\
$b$ & -0.1736 & -0.1642 & -0.1511 \\
$a_{\text {eff }}$ & 0.4373 & 0.3691 & 0.3147 \\
$b_{\text {eff }}$ & -0.1735 & -0.1640 & -0.1508 \\
\hline
\end{tabular}

of $H_{1}^{*}(\omega, r)$ (given in the Appendix), the final equation defining the $H_{2}^{*}(\omega, \omega, r)$ function is obtained. This nonhomogeneous ODE cannot be solved analytically, so it was solved numerically, by using the bvp4c solver in MATLAB. By using Eq. (21), the function $F_{2}^{*}(\omega, \omega, r)$ is obtained:

$$
F_{2}^{*}(\omega, \omega, r)=a_{e f f} H_{2}^{*}(\omega, \omega, r)+b_{e f f} H_{1}^{*^{2}}(\omega, r)
$$

where $b_{\text {eff }}$ is the effective second-order coefficient of the adsorption isotherm (Table 1). Finally, the second-order FRF with respect to the mean concentration in the particle $\langle q\rangle$ is obtained by using Eq. (22):

$$
F_{2}(\omega, \omega)=\frac{3}{R^{3}} \int_{0}^{R} r^{2} F_{2}^{*}(\omega, \omega, r) d r
$$

\section{Simulations of the FRFs and Characteristic Properties}

The first- and second-order FRFs derived in the previous section were simulated using MATLAB software for the system $\mathrm{CO}_{2}$ /zeolite $5 \mathrm{~A}$, by using an adsorption isotherm from literature [29]. In order to identify the characteristic properties of the FRFs, simulations for different steady-state pressures (20 mbar, $40 \mathrm{mbar}$, and $75 \mathrm{mbar}$ ) and different ratios of $D_{s} / D_{p}$ were performed. The pore diffusion coefficient was kept constant $D_{p}=10^{-7} \mathrm{~m}^{2} / \mathrm{s}$, and the surface diffusion coefficient was varied $D_{s}=\left(10^{-9}-10^{-7}\right) \mathrm{m}^{2} / \mathrm{s}$. The case $D_{s}=0$ $\left(D_{s} / D_{p}=0\right)$, which corresponds to pure pore diffusion mechanism, was also considered for comparison. The values of the adsorption isotherm coefficients $a, b, a_{e f f}$ and $b_{e f f}$ for all three considered pressures at $298 \mathrm{~K}$ are given in Table 2. Simulations were done for a particle radius $R=8.5 \mathrm{e}-04 \mathrm{~m}$ and particle porosity $\varepsilon=0.35$. The simulated FRFs are presented in the form of Bode plots (amplitude vs. frequency and phase vs. frequency).

In Figure 1 the first-order FRF (Figures 1(a) and 1(b)) and the second-order FRF (Figures 1(c) and 1(d)) for three different steady-state pressures and constant ratio $D_{s} / D_{p}=0.1$ are presented. From Figures 1(a) and 1(b) it can be seen that the amplitude of $F_{1}(\omega)$ has horizontal low frequency asymptote and a high frequency asymptote with a slope -0.5 and the phase of $F_{1}(\omega)$ has the low frequency asymptote zero, single inflection point, and the high frequency asymptote of $-\pi / 4$. As expected, the steady-state pressure influences only the amplitude curves. Although the pore-surface diffusion model has two rate constants (one corresponds to pore diffusion and the other to surface diffusion), they cannot be distinguished in the frequency window of the phase of $F_{1}(\omega)$ and a single inflection point of the phase might lead to misinterpretation of the pore-surface diffusion as a single rate process.

In contrast to the frequency spectrum of the firstorder FRF, where only one characteristic frequency (which corresponds to the inflection point of phase) is recognized, in the frequency spectrum of the second-order FRF (Figures $1(\mathrm{c})$ and $1(\mathrm{~d}))$, two characteristic frequencies, which correspond to the extreme values (one minimum and one maximum) of the phase, can be observed. The amplitude of $F_{2}(\omega, \omega)$ has a horizontal low frequency asymptote, two inflection points, and a high frequency asymptote with a slope -0.5 (Figure $1(c))$. The phase of $F_{2}(\omega, \omega)$ has a low frequency asymptote of $\pi$, a distinct minimum followed by a distinct maximum, and a high frequency asymptote of $3 \pi / 4$ (Figure $1(\mathrm{~d})$ ). As in the case of $F_{1}(\omega)$, the steady-state pressure influences only the amplitude but has no influence on the shape of the amplitude curve nor on the phase characteristics. The observed pattern of $F_{2}(\omega, \omega)$ with two extreme values of the phase indicates that two time constants (pore and surface diffusion) can be separated in the frequency window of $F_{2}(\omega, \omega)$.

In Figure 2 the first-order FRF (Figures 2(a) and 2(b)) and the second-order FRF (Figures 2(c) and 2(d)) for a constant steady-state pressure of $20 \mathrm{mbar}$ and different ratios of $D_{\mathrm{s}} / D_{\mathrm{p}}$ are given. The case $D_{\mathrm{s}}=0\left(D_{\mathrm{s}} / D_{\mathrm{p}}=0\right.$, dashed line $)$ corresponds to the pure pore diffusion. It can be seen from Figures 2(a) and 2(b) that $F_{1}(\omega)$ has the same shape for pure pore diffusion $\left(D_{\mathrm{s}} / D_{\mathrm{p}}=0\right)$ and pore-surface diffusion, which shows that no distinction between those two mechanisms can be made based on $F_{1}(\omega)$. However, Figures 2(c) and 2(d) show that $F_{2}(\omega, \omega)$ has qualitatively different shapes for pure pore and pore-surface diffusion. The amplitude of $F_{2}(\omega, \omega)$ for pure pore diffusion has one inflection while for pore-surface diffusion it has two inflections. The phase of $F_{2}(\omega, \omega)$ for $D_{s}=0$ has a single minimum, while for all other ratios $D_{\mathrm{s}} / D_{\mathrm{p}}$ a minimum followed by a maximum is observed. Both are shifted towards higher frequencies with increase of the contribution of surface diffusion. This result clearly shows that the shapes of the amplitude and phase of $F_{2}(\omega, \omega)$ can be used for reliable discrimination between poresurface and pure pore mechanisms. Moreover, comparison of the characteristic features of the amplitude and phase of $F_{2}(\omega, \omega)$ for pore-surface diffusion, pure pore diffusion (this work), and micropore diffusion model [18] in Table 3 proves that these characteristic features are distinct for each of these three mechanisms and can, therefore, be used for their discrimination. 


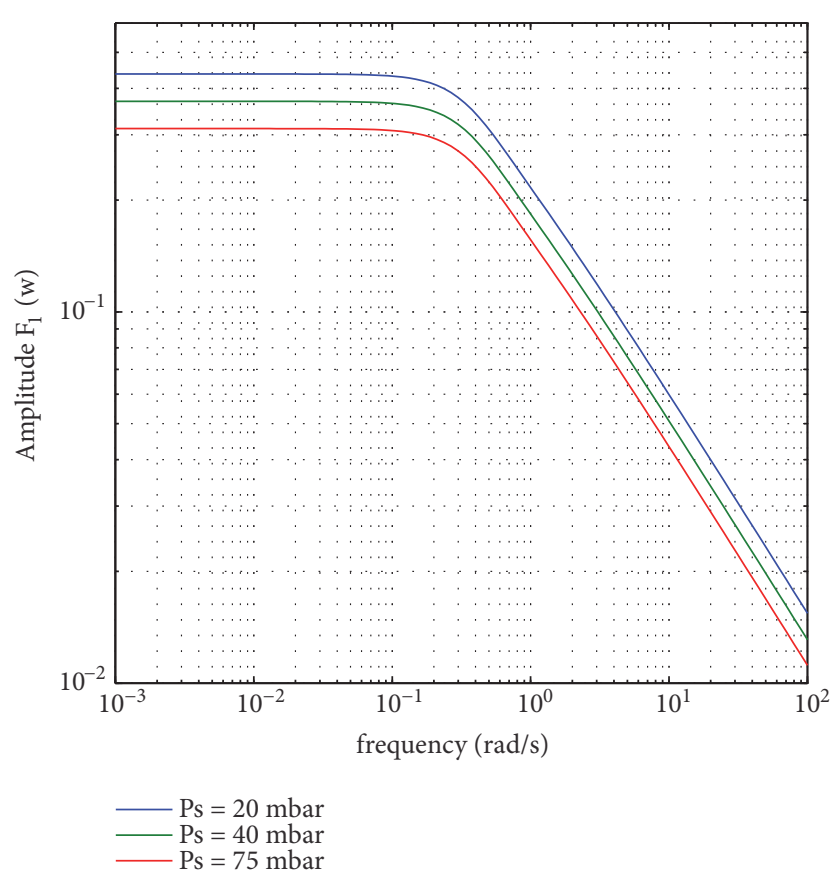

(a)

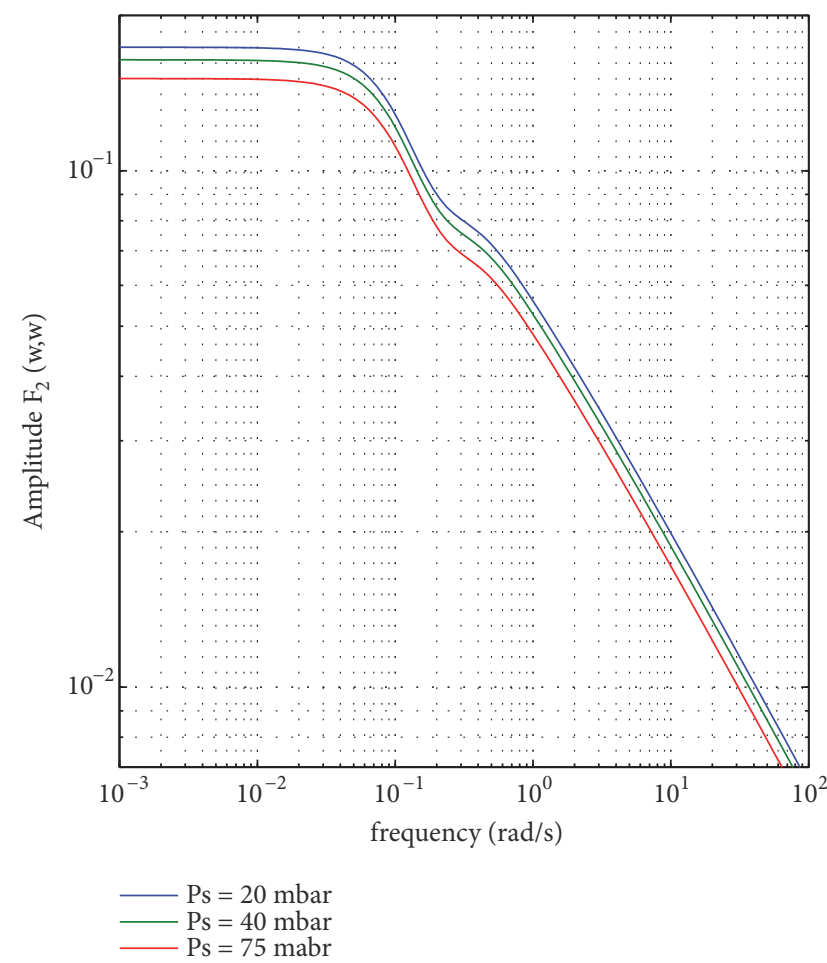

(c)

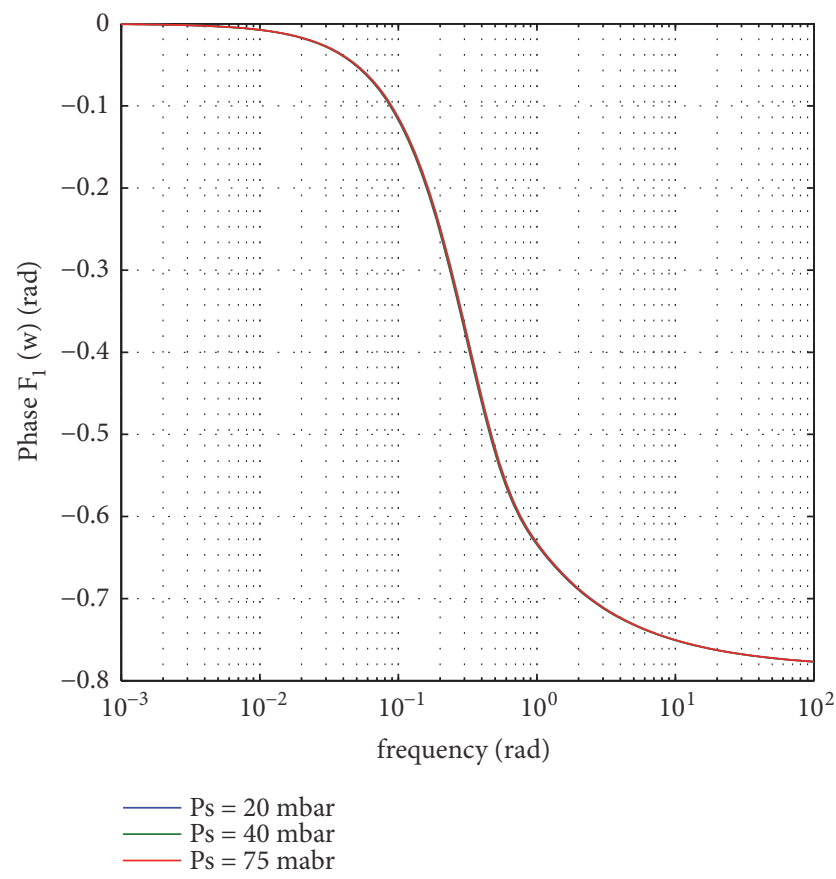

(b)

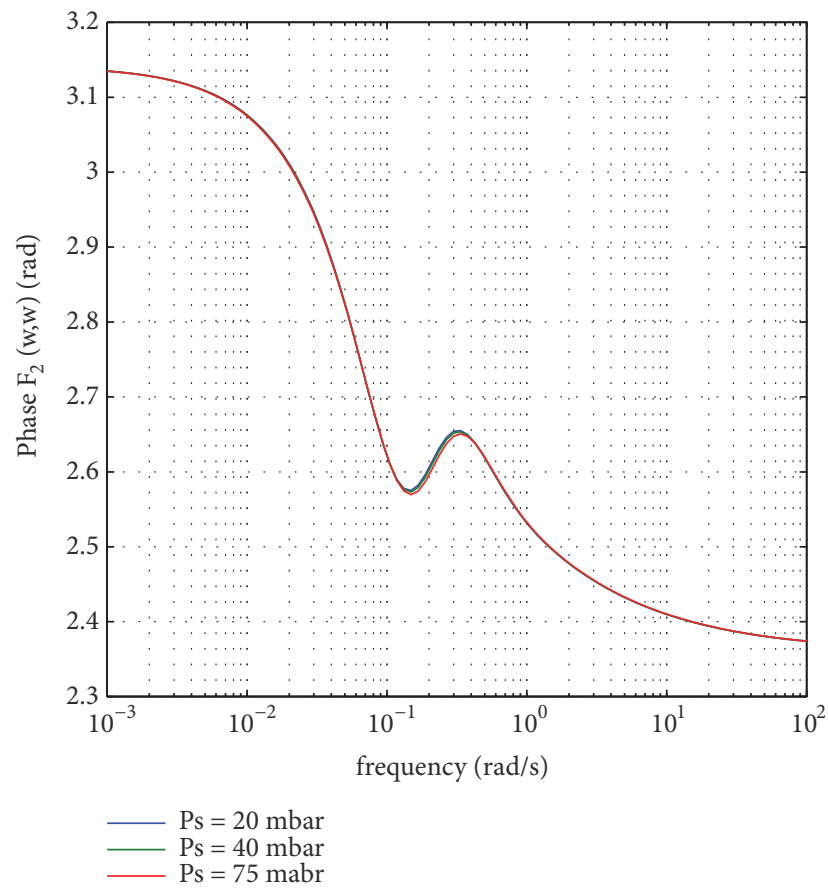

(d)

Figure 1: (a) Amplitude $F_{1}(\omega)$ vs. frequency; (b) phase $F_{1}(\omega)$ vs. frequency; (c) amplitude $F_{2}(\omega, \omega)$ vs. frequency; (d) phase $F_{2}(\omega, \omega)$ vs. frequency $\left(D_{p}=10^{-7} \mathrm{~m}^{2} / \mathrm{s} ; D_{s}=10^{-8} \mathrm{~m}^{2} / \mathrm{s}\right)$.

\section{Estimation of $D_{s}$ and $D_{p}$}

For the adsorption process governed by pore-surface diffusion, two time constants can be defined: (i) Pore diffusion time constant $\left(\tau_{\mathrm{p}}\right)$

$$
\tau_{p}=\frac{R^{2}}{D_{p}}\left[\frac{(1-\varepsilon) a+\varepsilon_{p}}{\varepsilon_{p}}\right]
$$




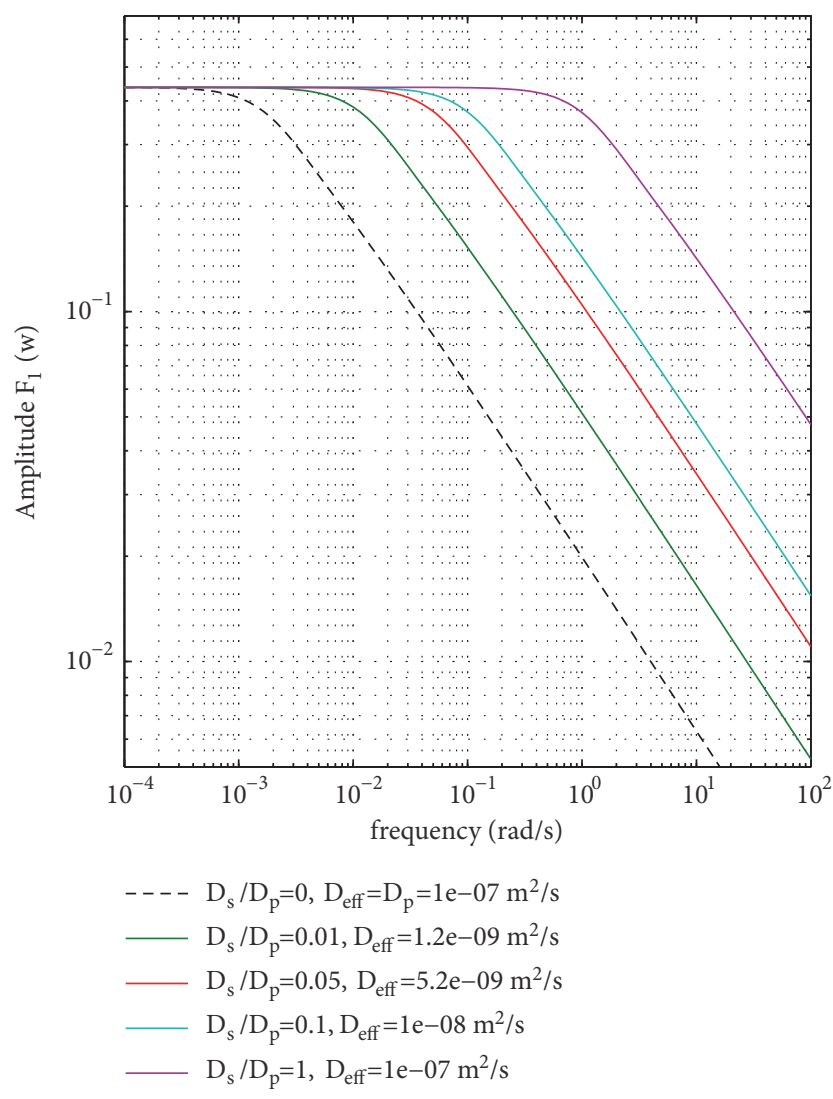

(a)

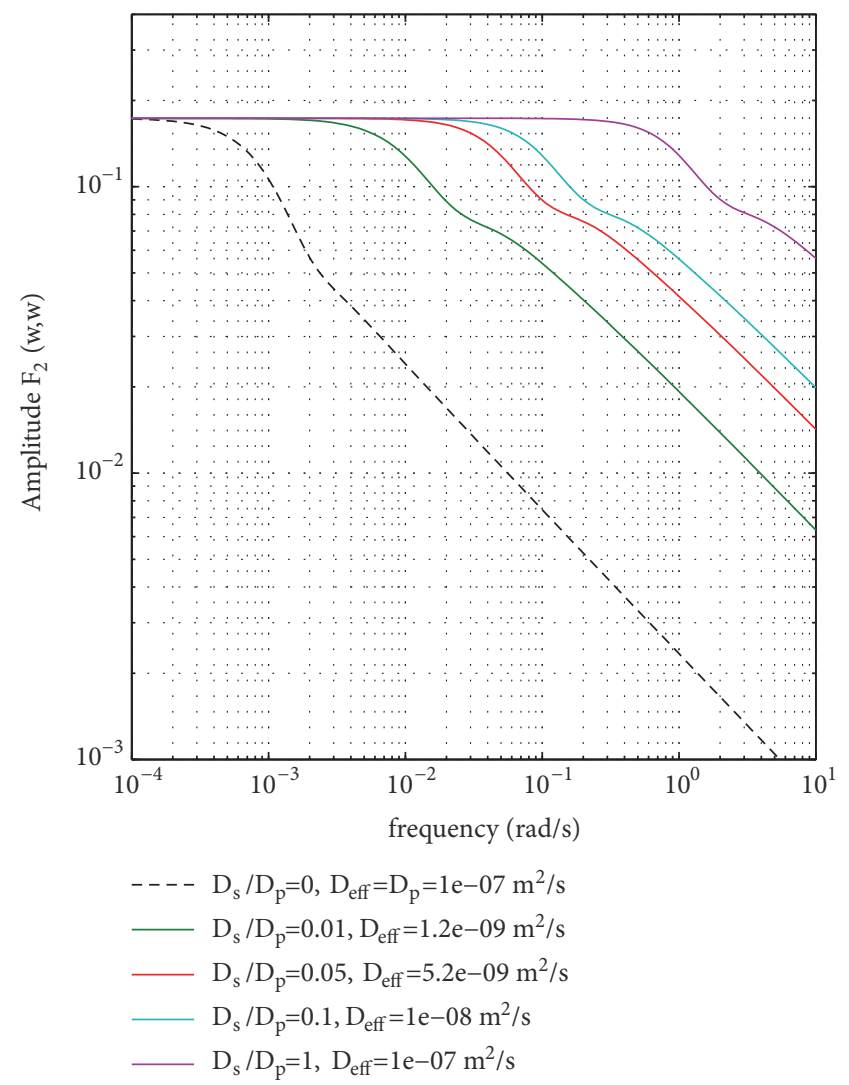

(c)
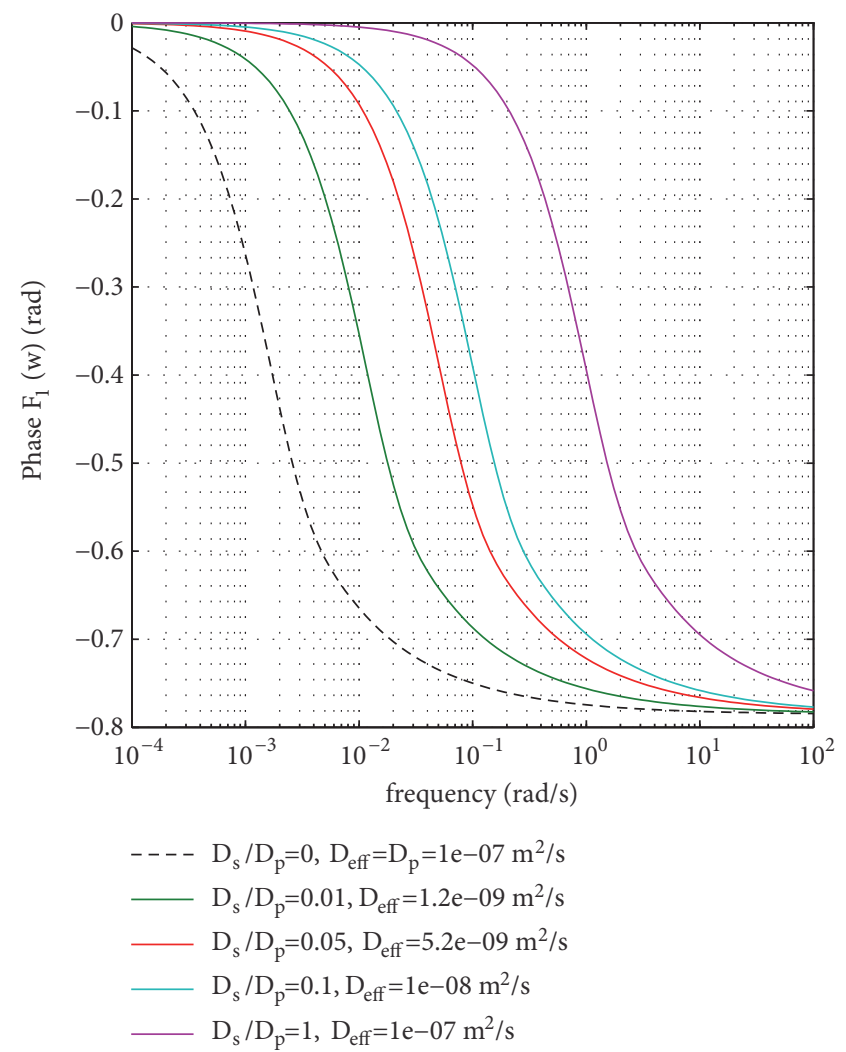

(b)

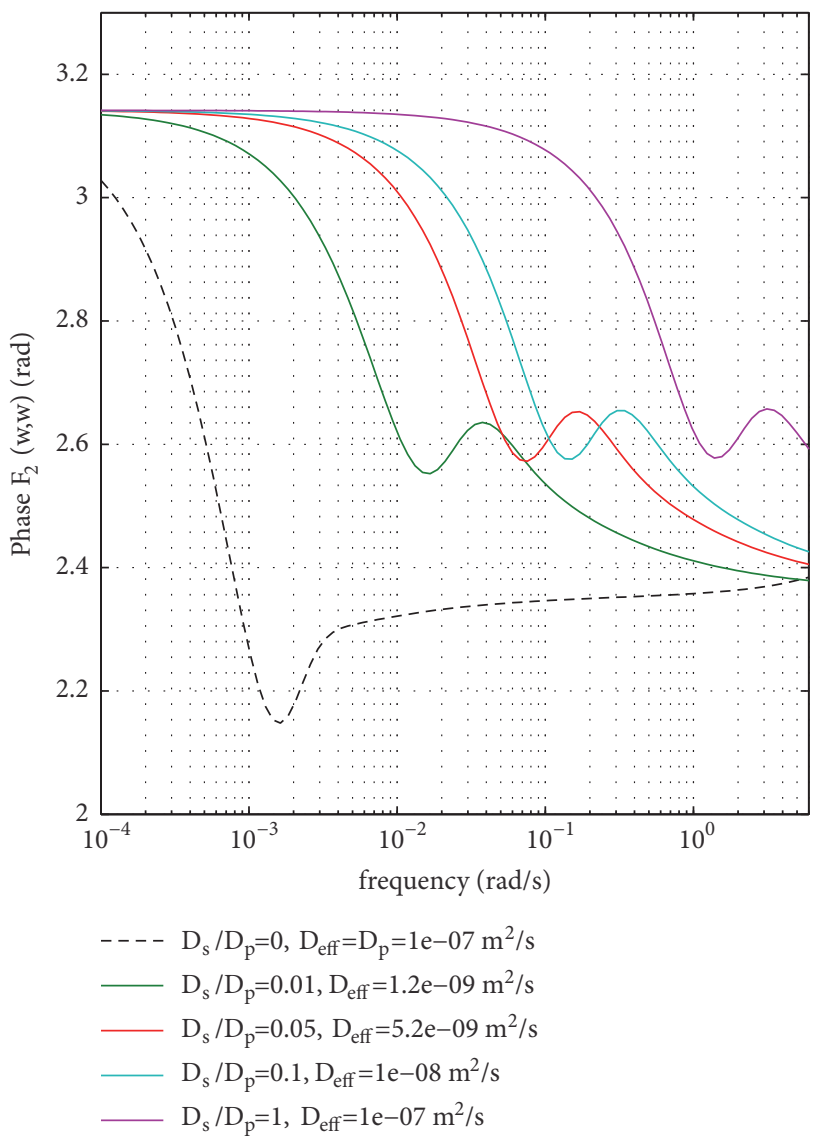

(d)

Figure 2: (a) Amplitude of $F_{1}(\omega)$ vs. frequency; (b) phase of $F_{1}(\omega)$ vs. frequency; (c) amplitude of $F_{2}(\omega, \omega)$ vs. frequency; (d) phase of $F_{2}(\omega, \omega)$ vs. frequency (steady-state pressure 20 mbar). 
TABLE 3: Characteristic features of $F_{2}(\omega, \omega)$ for different mechanisms (spherical geometry).

\begin{tabular}{lcc}
\hline & Amplitude $F_{2}(\omega, \omega)$ & Phase $F_{2}(\omega, \omega)$ \\
\hline Pore-surface diffusion & Two inflections & Minimum followed by maximum \\
Pure pore diffusion & One inflection & Single minimum \\
Micropore diffusion [18] & No inflections & Sigmoidal (single inflection) \\
\hline
\end{tabular}

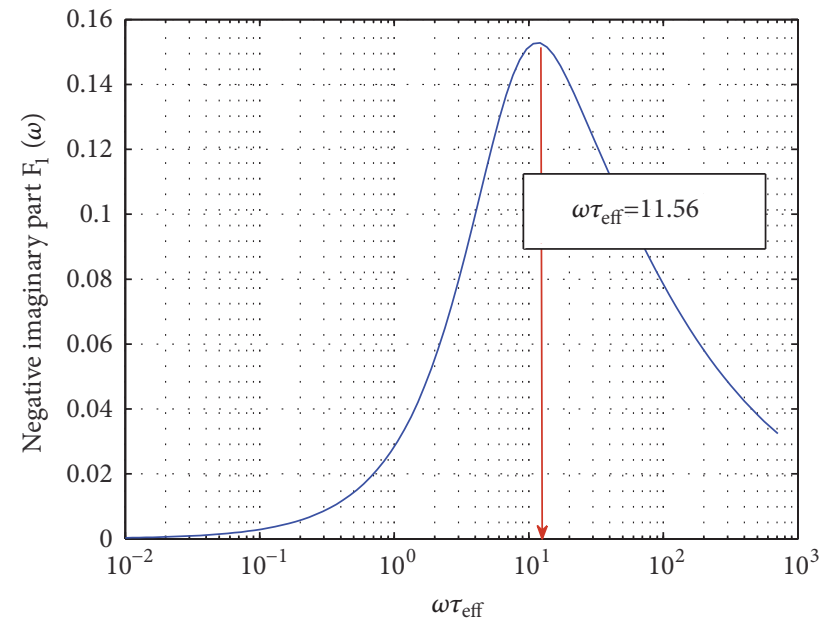

FIGURE 3: Negative imaginary part of $F_{1}(\omega)$ vs. $\omega \tau_{\text {eff }}[18]$.

(ii) Surface diffusion time constant $\left(\tau_{\mathrm{s}}\right)$

$$
\tau_{s}=\frac{R^{2}}{D_{s}}\left[\frac{(1-\varepsilon) a+\varepsilon_{p}}{(1-\varepsilon) a}\right]
$$

By taking into account the effective diffusion coefficient $D_{\text {eff }}$, defined by Eq. (26), it is also common to define the effective time constant $\left(\tau_{\text {eff }}\right)$ :

$$
\tau_{e f f}=\frac{R^{2}}{D_{e f f}}
$$

It is already known [18] that $\tau_{\text {eff }}$ can be estimated from the first-order FRF, actually from the locus of the maximum of negative imaginary part of $F_{1}(\omega)$ (Figure 3 ), by using the equation $\omega \tau_{e f f}=11.56$ (which is valid for spherical particles). $D_{\text {eff }}$ can then be calculated from Eq. (42).

In the previous section it was shown that the most remarkable feature of $F_{2}(\omega, \omega)$ is maximum right after the minimum of the phase of $F_{2}(\omega, \omega)$. In Figure 4 the phase of $F_{2}(\omega, \omega)$ (for different ratios $D_{s} / D_{p}$ ) is plotted vs. frequency $(\omega)$ (Figure 4(a)) and vs. dimensionless frequency, defined as frequency multiplied by surface diffusion time constant $\left(\omega \tau_{\mathrm{s}}\right)$ (Figure 4(b)). It turns out that, when plotted against $\omega \tau_{s}$, all curves overlap and the maximum corresponds to $\omega \tau_{\mathrm{s}}=25$. As a consequence, $\tau_{\mathrm{s}}$ can be estimated from the frequency at which the phase of $F_{2}(\omega, \omega)$ has a maximum, and $D_{s}$ can be further calculated from Eq. (41).

The procedure for estimation of $D_{s}$ and $D_{\mathrm{p}}$ from the experimental $F_{1}(\omega)$ and $F_{2}(\omega, \omega)$ can be summarized in three steps as follows:
(1) Identify the frequency at which the negative imaginary part of $F_{1}(\omega)$ has a maximum, $\omega_{\text {crit, } 1}$. Calculate $\tau_{\text {eff }}$ from the equation $\tau_{e f f}=11.56 / \omega_{\text {crit }, 1}$. Calculate $D_{\text {eff }}$ using Eq. (42) as $D_{\text {eff }}=R^{2} / \tau_{\text {eff }}$.

(2) Identify the frequency at which the phase of $F_{2}(\omega, \omega)$ has a maximum, $\omega_{\text {crit,2. }}$ Calculate $\tau_{s}$ from equation $\tau_{s}=$ $25 / \omega_{\text {crit }, 2}$. Calculate $D_{\mathrm{s}}$ using Eq. (41) as $D_{s}=\left(R^{2} / \tau_{s}\right)[((1-$ $\left.\left.\varepsilon) a+\varepsilon_{p}\right) /(1-\varepsilon) a\right]$.

(3) Calculate $D_{\mathrm{p}}$ using Eq. (26) as $D_{p}=\left(D_{e f f}[(1-\varepsilon) a+\right.$ $\left.\left.\varepsilon_{p}\right]-D_{s}(1-\varepsilon) a\right) / \varepsilon_{p}$.

\section{Conclusions}

In this work the Nonlinear Frequency Response analysis is applied for theoretical treatment of adsorption governed by parallel pore and surface diffusion, which is commonly encountered in commercial adsorbents. The derivation of the theoretical first- and second-order FRFs $\left(F_{1}(\omega)\right.$ and $\left.F_{2}(\omega, \omega)\right)$ for the pore-surface diffusion model and spherical particle geometry is given step by step. The derived FRFs relate the adsorbate concentration in the particle to the pressure of the surrounding gas (in the dimensionless form). For the first-order FRF the analytical solution was obtained, while the second-order FRF was obtained numerically. The derived FRFs were simulated by using the literature equilibrium data for $\mathrm{CO}_{2}$ /zeolite $5 \mathrm{~A}$. The varied parameters were the steadystate pressure and the ratio of surface to pore diffusion coefficient $\left(D_{\mathrm{s}} / D_{\mathrm{p}}\right)$. Unlike the first-order FRF, the secondorder FRF exhibits bimodal characteristics which reflect the dynamics of the parallel pore and surface diffusion processes. For all tested combinations of parameters, the amplitude and phase characteristics of $F_{2}(\omega, \omega)$ show the same patterns: two inflections of the amplitude and two extrema (a minimum followed by a maximum) of the phase. These features enable clear distinction of the pore-surface diffusion from pure pore diffusion and micropore diffusion mechanisms. Besides reliable mechanism identification, the second-order FRF, considered together with the first-order FRF, gives the possibility to estimate the separate values of the pore diffusion coefficient $\left(D_{\mathrm{p}}\right)$ and surface diffusion coefficient $\left(D_{\mathrm{s}}\right)$.

This work has both theoretical and practical importance. It gives a detailed procedure for the derivation of the firstand second-order FRFs which can be used for derivation of FRFs for other mechanisms. Further, the existing library of theoretical FRFs is extended. The potential of the nonlinear frequency response analysis in identification of the adsorption kinetic mechanism is proven. The practical importance lies in the fact that we offer a procedure for estimation of the separate values of the pore and surface diffusion coefficients which is very often needed in engineering praxis. 


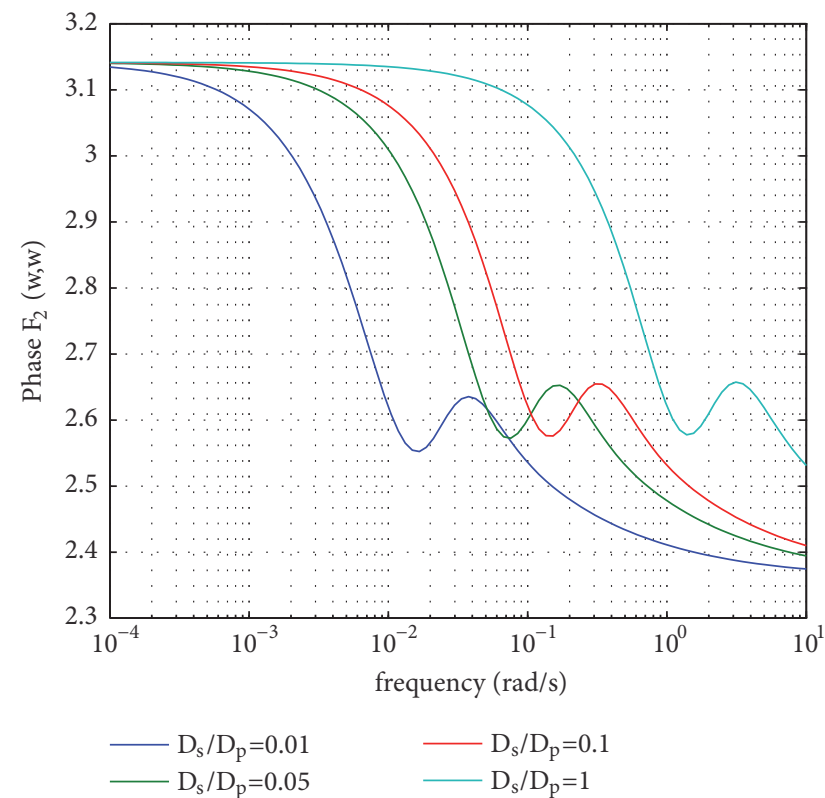

(a)

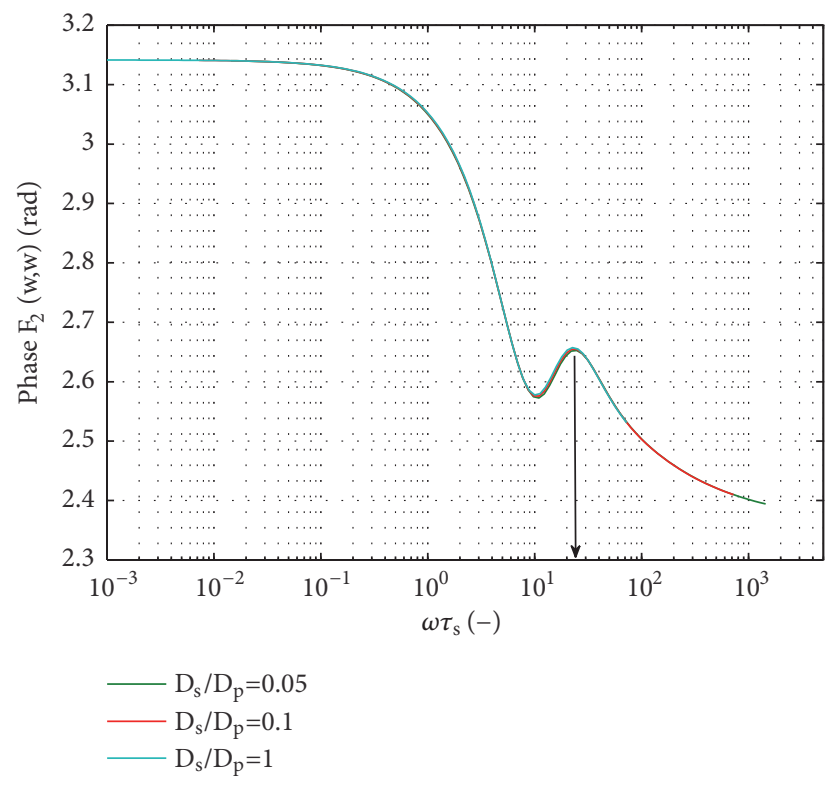

(b)

Figure 4: (a) Phase of $F_{2}(\omega, \omega)$ vs. frequency; (b) phase $F_{2}(\omega, \omega)$ vs. $\omega \tau_{\mathrm{s}}$.

\section{Appendix}

The first and the second derivatives of the function $H_{1}^{*}(\omega, r)$ defined by Eq. (29) are the following:

(i) The first derivative

$$
\begin{aligned}
\frac{d H_{1}^{*}(\omega, r)}{d r}= & \frac{R \sqrt{j \omega / D_{e f f}} \cosh \left(r \sqrt{j \omega / D_{e f f}}\right)}{r \sinh \left(R \sqrt{j \omega / D_{e f f}}\right)} \\
& -\frac{R \sinh \left(r \sqrt{j \omega / D_{e f f}}\right)}{r^{2} \sinh \left(R \sqrt{j \omega / D_{e f f}}\right)}
\end{aligned}
$$

(ii) The second derivative

$$
\begin{aligned}
\frac{d^{2} H_{1}^{*}(\omega, r)}{d r^{2}}= & \frac{j \omega R \sinh \left(r \sqrt{j \omega / D_{e f f}}\right)}{D_{e f f} r \sinh \left(R \sqrt{j \omega / D_{e f f}}\right)} \\
& -\frac{2 R \sqrt{j \omega / D_{e f f}} \cosh \left(r \sqrt{j \omega / D_{e f f}}\right)}{r^{2} \sinh \left(R \sqrt{j \omega / D_{e f f}}\right)} \\
& +\frac{2 R \sinh \left(r \sqrt{j \omega / D_{e f f}}\right)}{r^{3} \sinh \left(R \sqrt{j \omega / D_{e f f}}\right)}
\end{aligned}
$$

\section{Nomenclature}

A:

a:

$b:$

$c_{i}:$

$C_{i}$ :

$D_{p}:$

$D_{s}$ :

$D_{\text {eff }}$ :

$f:$

$F_{\mathrm{n}}\left(\omega_{1}, \omega_{2}, \ldots \omega_{\mathrm{n}}\right): \quad \mathrm{n}$-th order FRF relating $<q>$ to $p$ $F^{*}{ }_{\mathrm{n}}\left(\omega_{1}, \omega_{2}, \ldots \omega_{\mathrm{n}}\right): \mathrm{n}$-th order FRF relating $q(r)$ to $p$ $G_{\mathrm{n}}\left(\omega_{1}, \omega_{2}, \ldots \omega_{\mathrm{n}}\right):$ n-th order FRF relating $y$ to $x$ $H^{*}{ }_{\mathrm{n}}\left(\omega_{1}, \omega_{2}, \ldots \omega_{\mathrm{n}}\right): \mathrm{n}$-th order FRF relating $c_{i}(r)$ to $p$

$p:$

$P:$

$q_{i}:$

$Q_{i}:$

$R:$

$r:$

$t$ :

$x$

$y$
Amplitude of the input

First-order coefficient of the adsorption isotherm (Table 1) Second-order coefficient of the adsorption isotherm (Table 1)

Dimensionless sorbate concentration in the pore Sorbate concentration in the pore $\left(\mathrm{mol} / \mathrm{m}^{3}\right)$ Pore diffusion coefficient $\left(\mathrm{m}^{2} / \mathrm{s}\right)$ Surface diffusion coefficient $\left(\mathrm{m}^{2} / \mathrm{s}\right)$ Effective diffusion coefficient $\left(\mathrm{m}^{2} / \mathrm{s}\right)$ Dimensionless pressure Pressure (bar) Dimensionless sorbate concentration in the solid phase Sorbate concentration in the solid phase $\left(\mathrm{mol} / \mathrm{m}^{3}\right)$

Radius of the adsorbent particle (m) Spatial coordinate Time (s) Input Output. 


\section{Greek Letters}

$\varepsilon: \quad$ Porosity of the adsorbent particle

$\varepsilon_{p}$ : Modified porosity of the adsorbent particle

$\sigma$ : Shape factor

$\tau_{\mathrm{p}}$ : Pore diffusion time constant (Eq. (40))

$\tau_{\mathrm{s}}$ : Surface diffusion time constant (Eq. (41))

$\tau_{\text {eff }}:$ Effective diffusion time constant (Eq. (42))

$\omega:$ Frequency $(\mathrm{rad} / \mathrm{s})$.

\section{Data Availability}

The simulation data used to support the findings of this study are included within the article.

\section{Conflicts of Interest}

The authors declare that they have no conflicts of interest.

\section{Acknowledgments}

The financial support from the Ministry of Education and Science, Republic of Serbia, project number 172022, is gratefully acknowledged.

\section{References}

[1] D. M. Ruthven, Principles of Adsorption and Adsorption Processes, John Wiley, 1984.

[2] D. M. Ruthven, "Diffusion in type A zeolites: New insights from old data," Microporous and Mesoporous Materials, vol. 162, pp. 69-79, 2012.

[3] H. Ahn, J.-H. Moon, S.-H. Hyun, and C.-H. Lee, "Diffusion mechanism of carbon dioxide in zeolite $4 \mathrm{~A}$ and CaX pellets," Adsorption, vol. 10, no. 2, pp. 111-128, 2004.

[4] S. Brandani, "Analysis of the piezometric method for the study of diffusion in microporous solids: Isothermal case," Adsorption, vol. 4, no. 1, pp. 17-24, 1998.

[5] Y. H. Ma and C. Mancel, "Diffusion stidies of $\mathrm{CO}_{2}, \mathrm{NO}, \mathrm{NO}_{2}$, and $\mathrm{SO}_{2}$ on molecular sieve Zeolites by gas Chromatography," AIChE Journal, vol. 18, no. 6, pp. 1148-1153, 1972.

[6] W. Niessen and H. G. Karge, "Diffusion of p-xylene in single and binary systems in zeolites investigated by FTIR spectroscopy," Microporous Materials, vol. 1, no. 1, pp. 1-8, 1993.

[7] S. Kouva, J. Kanervo, F. Schüßler, R. Olindo, J. A. Lercher, and O. Krause, "Sorption and diffusion parameters from vacuumTPD of ammonia on H-ZSM-5," Chemical Engineering Science, vol. 89, pp. 40-48, 2013.

[8] T. Nguyen, M. Glorius, and C. Breitkopf, "A New approach to determine gas diffusion coefficients in porous solids by EIS: application for $\mathrm{NH}_{3}$ and $\mathrm{CO}_{2}$ adsorption on zirconia and zeolite type 5A," Advances in Mathematical Physics, vol. 2018, Article ID 5462659, 11 pages, 2018.

[9] L. Rodríguez-González, E. Rodríguez-Castellón, A. JiménezLópez, and U. Simon, "Correlation of TPD and impedance measurements on the desorption of NH3 from zeolite H-ZSM5," Solid State Ionics, vol. 179, no. 35-36, pp. 1968-1973, 2008.
[10] Y. Yasuda, "Frequency response method for investigation of gas/surface dynamic phenomena," Heterogeneous Chemistry Reviews, vol. 1, pp. 103-124, 1994.

[11] G. Onyestyák, D. Shen, and L. V. C. Rees, "Frequency-response studies of $\mathrm{CO}_{2}$ diffusion in commercial $5 \mathrm{~A}$ powders and pellets," Microporous Materials, vol. 5, no. 5, pp. 279-288, 1996.

[12] B. K. Sward and M. D. LeVan, "Frequency response method for measuring mass transfer rates in adsorbents via pressure perturbation," Adsorption, vol. 9, no. 1, pp. 37-54, 2003.

[13] T. J. Giesy, Y. Wang, and M. D. LeVan, "Measurement of mass transfer rates in adsorbents: New combined-technique frequency response apparatus and application to $\mathrm{CO}_{2}$ in $13 \mathrm{X}$ Zeolite," Industrial \& Engineering Chemistry Research, vol. 51, no. 35, pp. 11509-11517, 2012.

[14] T. M. Tovar, J. Zhao, W. T. Nunn et al., "Diffusion of $\mathrm{CO}_{2}$ in large crystals of Cu-BTC MOF," Journal of the American Chemical Society, vol. 138, no. 36, pp. 11449-11452, 2016.

[15] Y. Wang, C. S. Paur, and P. I. Ravikovitch, "New development in flow-through pressure-swing frequency response method for mass-transfer study: Ethane in ZIF-8," AIChE Journal, vol. 63, no. 3, pp. 1077-1090, 2017.

[16] V. Bourdin, P. Grenier, F. Meunier, and L. M. Sun, “Thermal frequency response method for the study of mass-transfer kinetics in adsorbents," AIChE Journal, vol. 42, no. 3, pp. 700712, 1996

[17] S. C. Reyes and E. Iglesia, "Frequency response techniques for the characterization of porous catalytic solids," in Catalysis, J. J. Spivey and S. K. Agarwal, Eds., vol. 11, pp. 51-92, Royal Society of Chemistry, London, UK, 2007.

[18] M. Petkovska, "Nonlinear frequency response method for investigation of equilibria and kinetics in adsorption systems," in Adsorption Systems in Finely Dispersed Particles: Micro Nano and Atto-Engineering, A. M. Spasic and J. P. Hsu, Eds., p. 283, CRC Taylor \& Francis, Boca Raton, FL, USA, 2006.

[19] M. Petkovska and D. D. Do, "Nonlinear frequency response of adsorption systems: Isothermal batch and continuous flow adsorbers," Chemical Engineering Science, vol. 53, no. 17, pp. 3081-3097, 1998.

[20] M. Petkovska and D. Do, "Use of higher-order frequency response functions for identification of nonlinear adsorption kinetics: Single mechanisms under isothermal conditions," Nonlinear Dynamics, vol. 21, no. 4, pp. 353-376, 2000.

[21] M. Petkovska, "Application of nonlinear frequency response to adsorption systems with complex kinetic mechanisms," Adsorption, vol. 11, no. 1, pp. 497-502, 2005.

[22] M. Petkovska, "Non-linear frequency response of nonisothermal adsorption controlled by micropore diffusion with variable diffusivity," Journal of the Serbian Chemical Society, vol. 65, no. 12, pp. 939-961, 2000.

[23] D. Brzić and M. Petkovska, "Nonlinear frequency response analysis of nonisothermal adsorption controlled by macropore diffusion," Chemical Engineering Science, vol. 118, pp. 141-153, 2014.

[24] D. Brzić and M. Petkovska, "Some practical aspects of nonlinear frequency response method for investigation of adsorption equilibrium and kinetics," Chemical Engineering Science, vol. 82, pp. 62-72, 2012.

[25] D. Brzić and M. Petkovska, "A study of applicability of nonlinear frequency response method for investigation of gas adsorption based on numerical experiments," Industrial \& Engineering Chemistry Research, vol. 52, no. 46, pp. 16341-16351, 2013. 
[26] D. Brzić and M. Petkovska, "Nonlinear Frequency Response measurements of gas adsorption equilibrium and kinetics: New apparatus and experimental verification," Chemical Engineering Science, vol. 132, no. 18, pp. 9-21, 2015.

[27] D. D. Weiner and J. F. Spina, Sinusoidal Analysis and Modeling of Weakly Nonlinear Circuits, Van Nostrand Reinhold Company, New York, NY, USA, 1980.

[28] M. Petkovska, "Nonlinear frequency response of nonisothermal adsorption systems," Nonlinear Dynamics, vol. 26, no. 4, pp. 351370, 2001.

[29] J. Tóth, "Uniform interpretation of gas/solid adsorption," Advances in Colloid and Interface Science, vol. 55, pp. 1-239, 1995. 


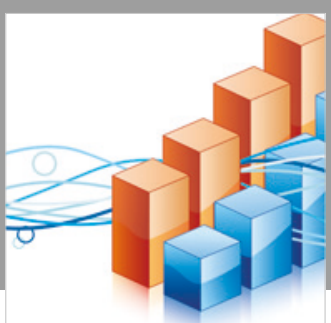

Advances in

Operations Research

\section{-n-m}
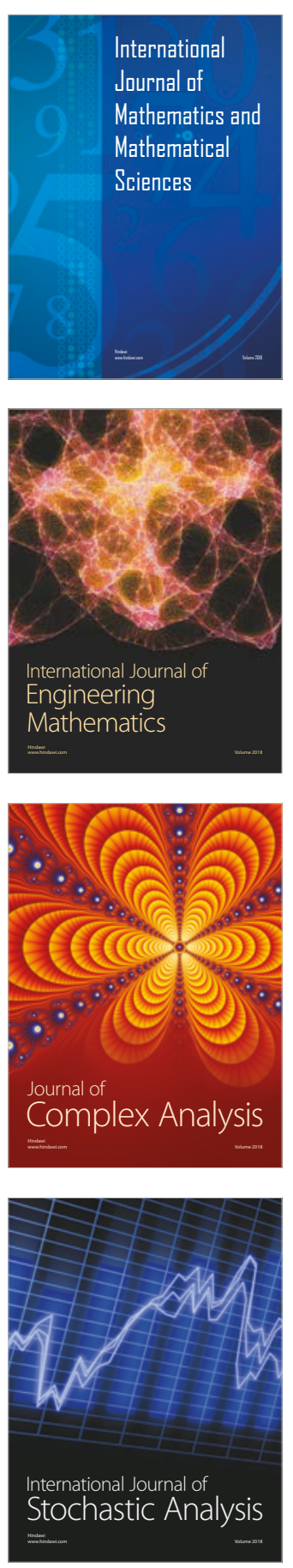
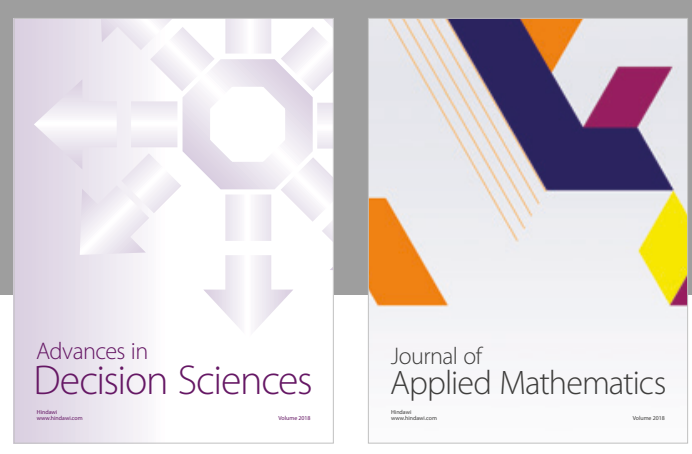

Journal of

Applied Mathematics
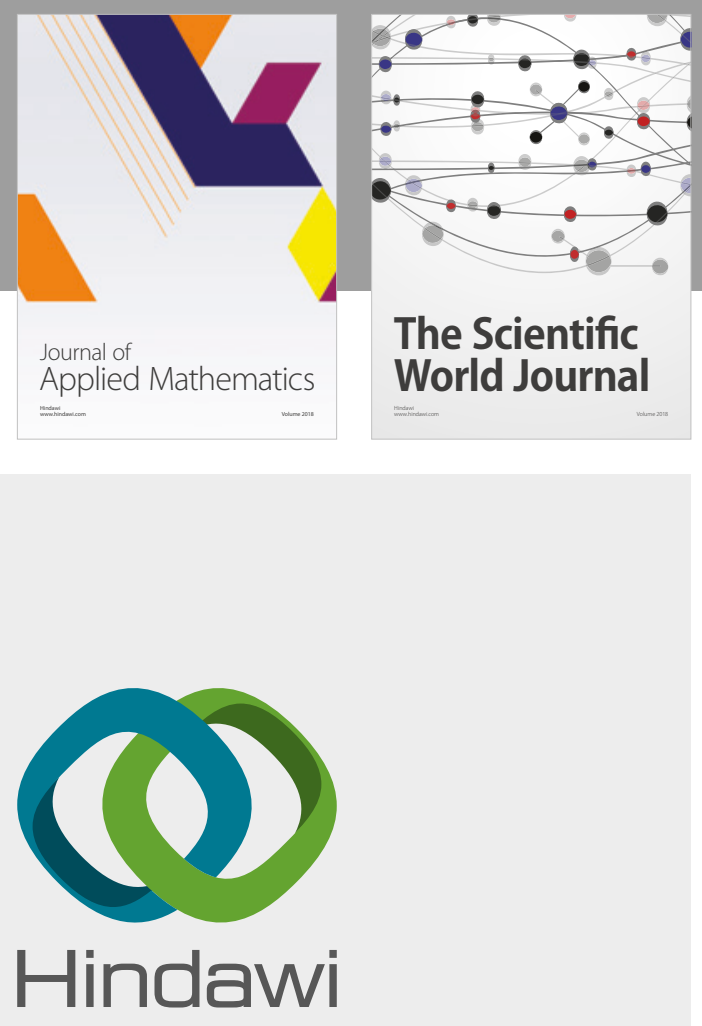

Submit your manuscripts at

www.hindawi.com

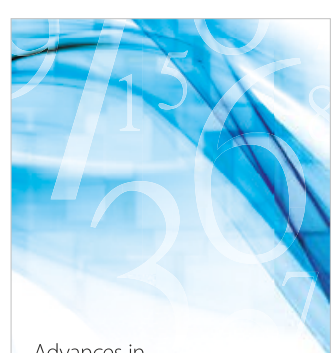

Advances in
Numerical Analysis
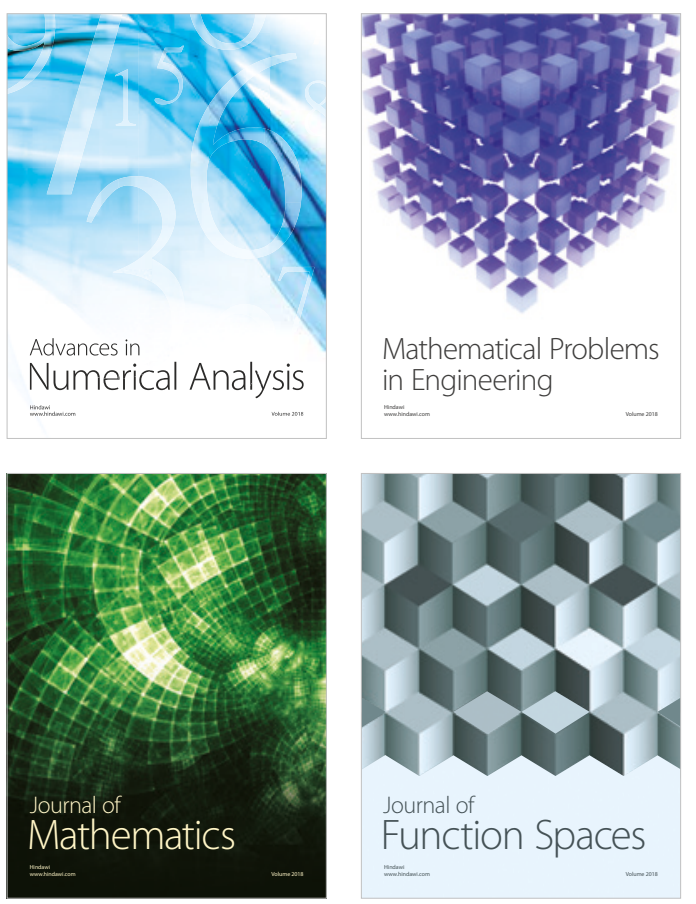

Mathematical Problems in Engineering

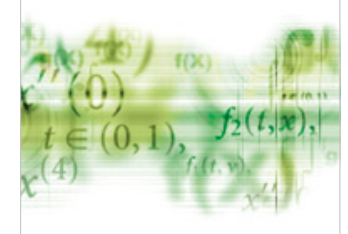

International Journal of

Differential Equations

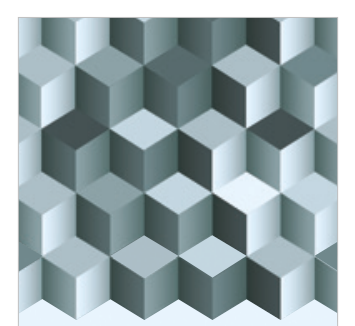

Journal of

Function Spaces

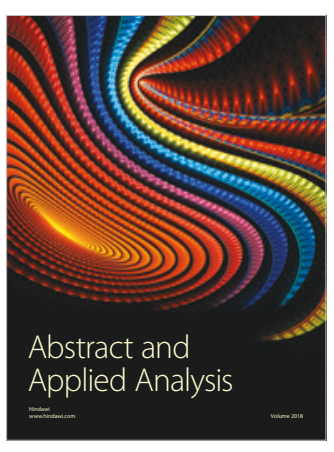

The Scientific

World Journal

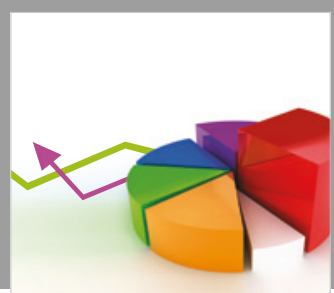

Journal of

Probability and Statistics
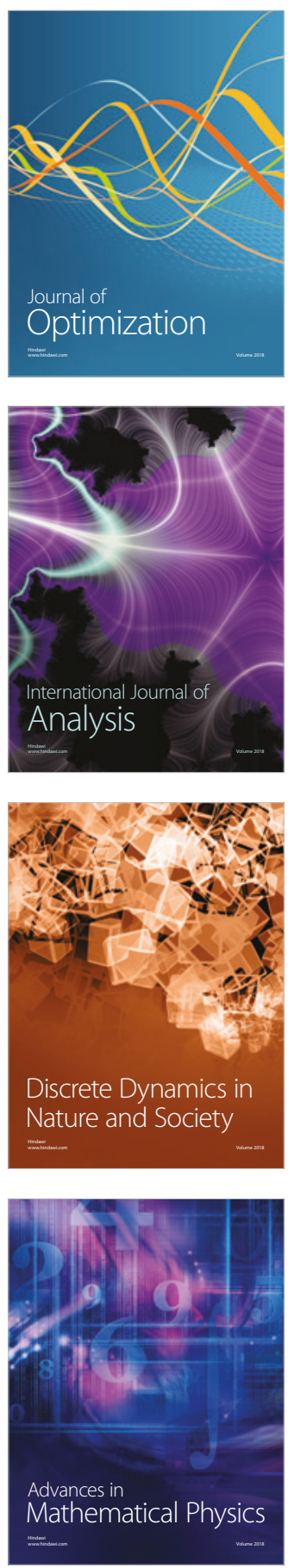